\title{
Identification of a novel candidate gene in the iron-sulfur pathway implicated in ataxia-susceptibility: human gene encoding HscB, a J-type co-chaperone
}

Received: 12 May 2003/ Accepted: 27 May 2003/Published online: 19 August 2003

(C) The Japan Society of Human Genetics and Springer-Verlag 2003

\begin{abstract}
Iron-sulfur proteins participate in a wide range of biochemical processes, including many that are central to mitochondrial electron transfer and energy metabolism. Mutations in two such proteins, frataxin and $\mathrm{ABCB} 7$, cause Friedreich ataxia and X-linked sideroblastic anemia with ataxia, respectively, rendering other participants in this pathway functional candidates for hereditary ataxia syndromes. Recently frataxin was shown to have an identical phylogenetic distribution with two genes and was most likely specifically involved in the same sub-process in iron-sulfur cluster assembly as one gene, designated $h s c B$, in bacteria. To set the stage for an analysis of the potential role of this candidate gene in human disease, we defined the human $H s c B$ cDNA, its genomic locus, and its pattern of expression in normal human tissues. The isolated human $H s c B$ cDNA spans $785 \mathrm{bp}$ and encodes a conserved 235-amino-acid protein, including a putative mitochondrial import leader. The $H s c B$ gene is found at chromosome $22 \mathrm{q} 11-12$ and is composed of six exons and five introns. Northern blot analyses of RNA from adult and fetal tissues defined a pattern of expression in mitochondria-rich tissues similar to that of frataxin, an expression pattern compatible with its implied role in mitochondrial energetics and related disease phenotypes.
\end{abstract}

Keywords Human gene $\cdot$ Hsc20 - Expression · Iron-sulfur pathway $\cdot$ Ataxia

The GenBank accession number for $H s c B$ is AY 191719

G. Sun · J. J. Gargus $(\bowtie) \cdot$ D. T. Ta $\cdot$ L. E. Vickery Department of Physiology and Biophysics, University of California, Sprague Hall, Rm 328, 839 Medical Sciences Court, Irvine, CA 92697-4034, USA E-mail: jjgargus@uci.edu

Tel.: + 1-949-8247702

Fax: + 1-949-8241762

J. J. Gargus

Division of Human Genetics, Department of Pediatrics, University of California, Irvine, CA, USA

\section{Introduction}

Iron-sulfur proteins participate in a wide range of biochemical processes including many that are central to mitochondrial electron transfer and energy metabolism. Several studies have provided the evidence that this class of proteins play an important role in the development of human neurological disease, particularly ataxia. Loss of function alleles of frataxin produce functional mitochondrial defects in patients with Friedreich ataxia (MIM 229300) (Patel et al. 2001), and nulls of the yeast orthologue, $Y F H 1$, display a respiratory-deficient phenotype and a defect in Fe-S cluster formation (Muhlenhoff et al. 2002). Other hereditary ataxia syndromes, including specifically X-linked sideroblastic anemia with ataxia (MIM 300135), caused by mutations in the ABCB7 ATP-binding cassette protein (Bekri et al. 2000), seem to share these features (Kaplan 2002). Several genes encoding proteins involved in the biogenesis of iron-sulfur proteins have been identified in bacteria and yeast, and structural and functional studies of their encoded proteins are underway. Thus, their human homologues become richly annotated functional candidate genes in heritable ataxia syndromes.

A recent study found that frataxin showed an identical phylogenetic distribution with only two genes and most likely was specifically involved in the same subprocess in iron-sulfur cluster assembly as one of these genes, designated $h s c B$ in bacteria and $J A C l$ in yeast (Huynen et al. 2001). The $h s c B$ gene in bacteria encodes a 20-kDa J-type co-chaperone, designated Hsc20, that functions with the hsp70-class molecular chaperone HscA/Hsc66 (Seaton and Vickery 1994; Kawula and Lelivelt 1994; Vickery et al. 1997). In vitro studies have shown that $\mathrm{HscB} / \mathrm{Hsc} 20$ regulates interactions between the Fe-S cluster assembly protein IscU and the $\mathrm{HscA} /$ Hsc66 chaperone (Hoff et al. 2000; Silberg et al. 2001; Hoff et al. 2002). Homologues of the bacterial $h s c B$ gene are found in eukaryotes, and the homologue in Saccharomyces cerevisiae, $J A C 1$, is a nuclear gene whose 
protein product is targeted to mitochondria (Strain et al. 1998; Kim et al. 2001; Lutz et al. 2001; Voisine et al. 2001). The $J A C l$ gene is essential, and mutations in the gene have been found to cause defects in iron-sulfur protein activities, mitochondrial iron accumulation, and reduced mitochondrial respiratory activity (Strain et al. 1998; Kim et al. 2001; Lutz et al. 2001; Voisine et al. 2001). Because the phylogenetic distribution of $h s c B /$ $J A C 1$ is identical to that of frataxin, both being repeatedly lost or transferred in concert, it is likely that Hsc20/Jaclp and frataxin are involved in similar highly conserved cellular processes (Huynen et al. 2001). Furthermore, the finding that mutations in both genes in yeast produce similar defects in Fe-S cluster formation and in mitochondrial respiration and that these processes are implicated in the pathogenic mechanism of ataxia syndromes suggests that mutations in HscB may likewise produce disease susceptibility. To set the stage for an analysis of the potential role of the $h s c B / J A C l$ gene in human disease, we defined the human $H s c B$ cDNA, the map position of its locus, the intron-exon boundaries within the locus, its pattern of expression in normal human tissues, and place this in the context of structural and functional studies of its model system homologues.

\section{Materials and methods}

\section{The $H s c B$ cDNA}

A similarity search for the human $H s c B$ cDNA was carried out using the Escherichia coli Hsc20 protein sequence with the tBLASTN program against the human EST database (http:// www.ncbi.nlm.nih.gov/BLAST). The search revealed several significant alignments with several EST clones (AI739537, AI796137, AW250733, BE279981, BE782421). The IMAGE clone number 2391082 (AI739537) was obtained and the complete sequence of its insert obtained sequencing both strands using T7 and T3 primers that bind sites flanking the insert. Potential reading frames were identified using Translate Tool (available at http://us.expasy.org/ tools/dna.html).

\section{Defining the genomic locus of $H s c B$}

The BLASTN program was used with the defined $H s c B$ cDNA sequence against the non-redundant human genomic database (http://www.ncbi.nlm.nih.gov/BLAST) yielding two positive entries (AL117330 and AL023494). The identification of exons and exon-intron junctions was carried out by merging the cDNA sequence with these genomic sequences.

Defining the $H s c B$ gene expression pattern using Northern blot analysis

Human multiple tissues Northern (MTN) and human fetal multiple tissues Northern blots were obtained from CLONTECH. Each MTN blot lane contains $2 \mu \mathrm{g}$ of purified poly $\left(\mathrm{A}^{+}\right)$RNA. A 231-bp fragment of $H s c B$ cDNA (complementary to messenger sequence 472-703 bp downstream from the initiation codon) was obtained by PCR and purified. This fragment was labeled with $\alpha-{ }^{32}$ P-UTP $(3,000 \mathrm{Ci} / \mathrm{mmol})$ by in vitro transcription labeling using a MAXIscript kit (Ambion, Austin, Texas, USA). Blots were hybridized with the labeled probe, washed and exposed to X-ray film (Fuji) at $-70{ }^{\circ} \mathrm{C}$ with two intensifying screens. Blots were stripped with $0.5 \%$ SDS at $95{ }^{\circ} \mathrm{C}$, and re-hybridized with labeled $\beta$-actin cDNA probe as a standard.

\section{Results}

The Escherichia coli Hsc20 protein sequence was used to carry out a similarity search in the human EST database, revealing several significant alignments. Sequencing the complete insert of one such clone, the IMAGE human cDNA clone number 2391082 (AI739537), revealed the full-length sequence of human $H s c B$ cDNA. This sequence has been deposited with GenBank and given accession number AY191719. It has a length of $785 \mathrm{bp}$ and encodes a predicted protein of 235 amino acids, with $34 \%$ and $29 \%$ overall identity to the E. coli $\mathrm{Hsc} 20$ and yeast JAC1 homologues, respectively (Fig. 1), with higher identity in core domains (see below). The conservation of this sequence through distant phylogeny suggests a conserved functional role in a core cellular process.

The two genomic entries containing portions of the human $H s c B$ sequence (AL117330 and AL023494) map to chromosome 22q11-12, and together include nearly the complete sequence of $H s c B$. The $H s c B$ gene has six exons and five introns spanning $15.3 \mathrm{~kb}$. The exon and intron sizes and the exon-intron junction sequences are shown in Table 1, and the organization of the $H s c B$ gene is shown in Fig. 2. Two monogenic familial ataxia syndromes have been mapped to chromosome 22q, spinocerebellar ataxia type 10 (SCA10; MIM 603516) and mitochondrial neurogastrointestinal encephalopathy syndrome (MNGIE; MIM 603041), but both are significantly telomeric to $H s c B$ and genes for both have already been assigned (Matsuura et al. 2000; Nishino et al. 1999).

The predicted protein encoded by the human $H s c B$ gene has features consistent with those determined for the E. coli Hsc20 homologue. The crystal structure of $E$. coli Hsc20 revealed that the protein consists of distinct $\mathrm{N}$ - and $\mathrm{C}$-terminal domains that make contact through an extensive hydrophobic interface (Cupp-Vickery and Vickery 2000). The N-terminal domain (residues $1-75)$ is folded into a compact $\alpha$-topology similar to the evolutionarily conserved J-protein co-chaperone motif required for chaperone activation (Kelley 1998). The J-domain is connected by a short loop to the C-terminal domain (residues 84-171), which is folded into a compact three-helix bundle (Cupp-Vickery and Vickery 2000). Residues 72-145 of human HscB are 43\% identical to the J-domain region of E. coli Hsc20, and the His-Pro-Asp J-domain signature motif is found at positions 102-104. Residues $154-235$ are $30 \%$ identical to the C-terminal domain of E. coli $\mathrm{Hsc} 20$. The predicted human protein has an $\mathrm{N}$-terminal extension of 71 residues not present in the bacterial protein. This extension is enriched in positively charged, hydroxylated, and hydrophobic residues consistent with a role as 
a mitochondrial-targeting signal (Pfanner 2000; Pfanner and Wiedemann 2002).

The exon structure of the human $H s c B$ gene appears to reflect the functional domains of the protein (Fig. 2). Exon 1 encodes the putative mitochondrial presequence (residues 1-71) as well as several amino acids presumed

a

aggccgccggccagatgtggcgggggagagccggggetttgctccgggtgtgggggttttgg 62 $\begin{array}{llllllllllllllllllllll}M & W & R & G & R & A & G & A & L & L & R & V & \text { W } & G & \text { F } & \text { W } & 16\end{array}$ ccgacaggggttcccagaaggagaccgctaagctgcgatgctgcgtcgcaggcgggaagc 122 $\begin{array}{lllllll}P & T & G & V\end{array}$ aattatccccgctgttggagctgcggcggcccatggggcccegggcgggaggacaggttc $\begin{array}{lllllllllllllllllllll}N & Y & P & R & C & W & S & C & G & G & P & W & G & P & G & R & E & D & R & F\end{array}$ ttctgcccacagtgccgagcgctgcaggcacctgaccccactcgagactacttcagcctt

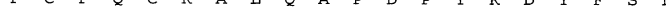
atggactgcaaccgttccttcagagttgatacagegaagetccagcacaggtaccagcaa

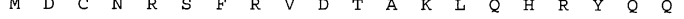
ctgcagcgtcttgtccacccagatttcttcagccagaggtctcagactgaaaaggacttc

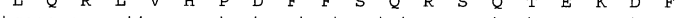
tcagagaagcattcgaccetggtgaatgatgcctataagaccctcctggecccctgagc $\begin{array}{llllllllllllllllllll}S & E & K & H & S & T & L & V & N & D & A & Y & K & T & L & L & A & P & L & S\end{array}$ agaggactgtaccttctaaagctecatggaatagagattcctgaaaggacagattatgaa

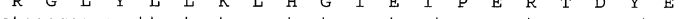
atggacaggcaattcctcatagaaataatggaaatcaatgaaaactcgcagaagctgaa

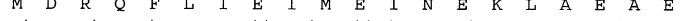
agtgaagctgccatgaaagagattgaatccattgtcaaagctaaacagaaagaatttact

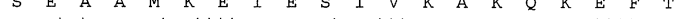
gacaatgtgagcagtgcttttgaacaagatgactttgaagaagccaaggaaattttgaca D $N$ V S S S A aagatgagatactttcaaatatagaagaaaagatcaagttaaagaagattcccctttaa

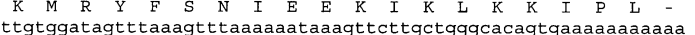

b aaa

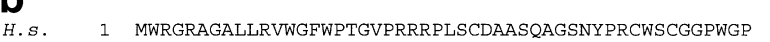

S.c. 1 MLKYLVQRRFTSTFYELFPKTFPKKLPIWTIDQSRLRKEYRQLQAQ

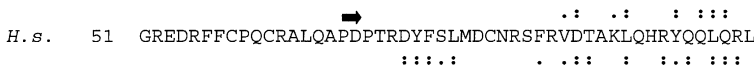

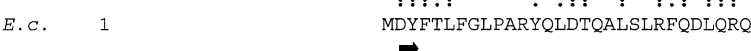

S.C. 47 HHPDMAQQGS--- --EQSSTLNQAYHTLKDPLRRSQYMLKLLRNIDLTQ

-

H.s. 101 VHPDFFSQRSQTEKDFSEKHSTLVNDAYKTLLAPLSRGLYLLKLH-GIEIP

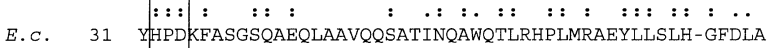

S.C. 91 EQTSNEVTTSDPQLLLKVLDIHDELSQMDDEAGVKLLEKQNKERIQDIEAQLG 143

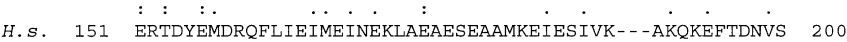

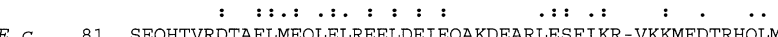

SEQHVDTAFMEQLELREELDE I EQAKDEARLESF I KR - VKKMFDTRHOLM

S.c. 144 QCYNDKDYAAAVKLTVELKYWYNLAKAFKDWAPGKQLEMNH 184

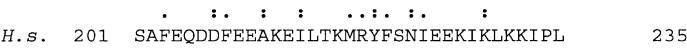

E.c. 133 VEQLDNETWDAAADTVRKLRFLDKLRSSAEQLEEKLLDF 171

Fig. 1a, b cDNA and deduced amino acid sequence of human HscB. a Sequence of IMAGE clone no. 2391082 and deduced amino acid sequence. b Alignment of the deduced amino acid sequences of HscB proteins from Homo sapiens (H.s.), S. cerevisiae (S.c.) and E. coli (E.c.). Sequence identities (:) and similarities (.) are indicated. The J-protein signature sequence, His-Pro-Asp, is boxed, and the N-termini of the mature forms of $S$. cerevisiae mitochondrial Jaclp and $E$. coli $\mathrm{HscB}$ are indicated by arrows to occur at the N-terminus of the J-domain (residues 7179). Exons 2 and 3 encode the core of the J-domain, encompassing residues $80-111$ and $112-143$, respectively. Exon 4 encodes residues 144-189 that form the short loop connecting the $\mathrm{J}$ - and $\mathrm{C}$-domains and the initial portion of the C-domain. Exons 5 and 6 encode residues 190-235 and comprise the majority of the C-domain.

The structure and implied function of human $\mathrm{HscB}$ suggest a mitochondrial role for the protein. In order to determine if the pattern of expression of human $H s c B$ was compatible with this hypothesis, Northern blot analyses were carried out using labeled $H s c B$ to probe poly $\left(\mathrm{A}^{+}\right)$RNA from multiple adult and fetal human tissues. Figure 3 shows that the highest levels of the mRNA for $H s c B$ are found in liver, muscle and heart, with very low levels elsewhere. This pattern of expression in mitochondria-rich tissues is similar to that observed for human frataxin, both sharing very low CNS expression levels (Campuzano et al. 1996). Unlike frataxin, however, the transcript size of human $H s c B$ varied depending on tissue type. The highest level of human $H s c B$ expression was found in adult and fetal liver in which a transcript of $\sim 2.5 \mathrm{~kb}$ was observed. Adult heart and skeletal muscle also displayed significant expression, and two transcripts, $\sim 1.4 \mathrm{~kb}$ and $\sim 6 \mathrm{~kb}$, were observed. The higher level of expression of $H s c B$ in these mitochondria-rich, metabolically active tissues is compatible with the implied role of human $\mathrm{HscB}$ in iron-sulfur cluster formation, interaction with frataxin and mitochondrial energetics, and is consistent with the observed N-terminal extension mitochondrial import sequence. The presence of different transcript sizes in different tissues may suggest alternatively spliced variants, possibly reflecting tissue-specific regulation.

\section{Discussion}

The predicted human $\mathrm{HscB}$ protein is similar in its general features to the homologous bacterial (Hsc20/ $\mathrm{HscB})$ and yeast (Jaclp) co-chaperones. Each is predicted to be approximately $20-\mathrm{kDa}$ in the mature form, and each has a highly conserved $\mathrm{N}$-terminal $\mathrm{J}$-domain linked to a less well-conserved $\mathrm{C}$-terminal region. The J-domains of Hsc20 and Jaclp have been implicated in interactions with their Hsp70 partners, and it is likely that this region of human HscB carries out the

Table 1 Sequences at the exon-intron boundaries and the sizes of exons and introns of human $H s c B$. Intron sequence is shown in lowercase and exon sequence in uppercase; tss translation start site

\begin{tabular}{lllllll}
\hline Exon no. & Exon size(bp) (coding region) & Splicing acceptor & $5^{\prime}$-Exon junction & 3'-Exon junction & Splicing donor & Intron size (bp) \\
\hline 1 & 326 & & tss & -TTATGGACTG & gtacgagcga- & 1,551 \\
2 & 97 & -ttccttccag & CAACCGTTCC- & -GAG GTCTCAG & gtagcttatt- & 636 \\
3 & 95 & -ccactaacag & ACTGAAAAGG- & -ACCTTCTAAG & gtgatttccc- & 1,160 \\
4 & 139 & -atagctaaag & CTCCATGGAA- & -ATTGTCAAAG & gtgaaagata- & $>5,332$ \\
5 & 49 & -tctt ttcag & CTAAACAGAA- & -TTTGAACAAG & gtactttctt- & 5,788 \\
6 & 92 & -tctattcag & ATGACTTTGA- & -poly(A) & & \\
\hline
\end{tabular}




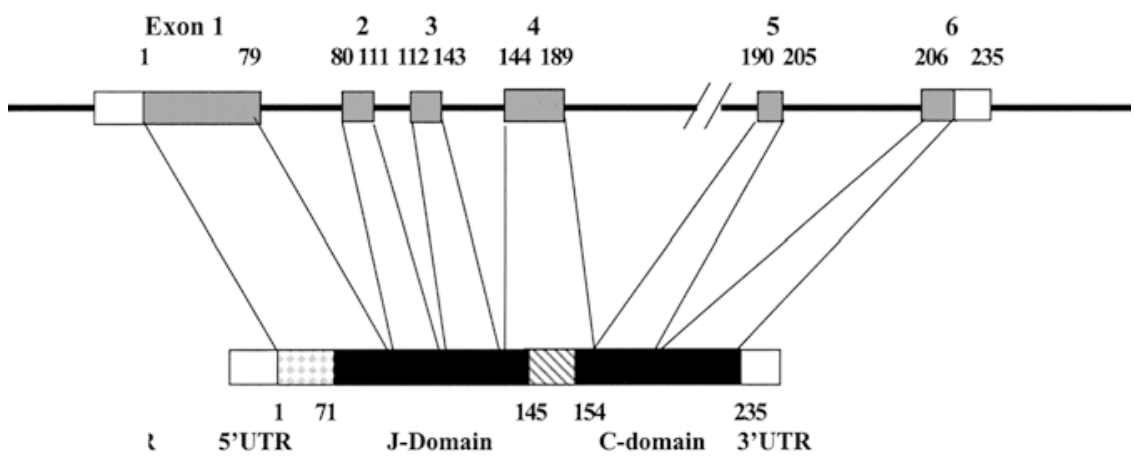

Fig. 2 Genomic organization of $H s c B$. The gene structure is represented at the top, with introns indicated as a thin line and exons indicated as a box, which is labeled above with the exon number and the amino acid residue positions encoded. The structure of the transcript is presented below, with the translated sequences shown as filled boxes and untranslated sequences as open boxes. Regions encoding the predicted $\mathrm{J}$ - and $\mathrm{C}$-domains of the protein are indicated by black boxes, with residue numbers shown below; the proposed mitochondrial signal sequence is indicated by a stippled box

orthologous function. The role of the $\mathrm{C}$-terminal region of $\mathrm{Hsc} 20 / \mathrm{Jacl} \mathrm{p}$ has not been defined. C-terminal regions of Hsp40 class J-type co-chaperones have been implicated in peptide substrate binding, but the C-terminal domain of $\mathrm{HscB}$ lacks structural features found in the larger Hsp40 proteins. Huynen and co-workers have suggested the possibility that frataxin may fill the role of the substrate-binding regions missing from $\mathrm{HscB}$ and that the co-evolution of frataxin with $\mathrm{HscB}$ may reflect the interaction of a J-type co-chaperone and a substrate binding protein required for its function (Huynen et al. 2001). While there are no experimental results to support this hypothesis, HscB and human frataxin both have conserved regions on the protein surface that may play a role in protein-protein interactions (Cupp-Vickery and Vickery 2000; Dhe-Paganon et al. 2000); several of these residues are found to be altered in pathogenic point mutant alleles of human frataxin (Dhe-Paganon et al. 2000). In addition, studies with recombinant human frataxin have shown that frataxin is able to sequester iron in vitro (Cavadini et al. 2002), and a role in iron binding could provide a possible functional link to $\mathrm{HscB}$ in iron-sulfur cluster biogenesis.

HscB shares many functional aspects in common with frataxin suggesting a common role in cellular physiology and perhaps additionally in pathophysiology. The features of mitochondrial expression and relatively low CNS expression observed for $\mathrm{HscB}$ have previously been recognized for frataxin (Campuzano et al. 1996) as has the shared energy-deficient phenotype of mutants of both in model organisms and their implied shared role in $\mathrm{Fe}-\mathrm{S}$ cluster biosynthesis (Strain et al. 1998; Muhlenhoff et al. 2002; Kim et al. 2001; Lutz et al. 2001; Voisine et al. 2001). The relatively low level of expression in the CNS the two share may render this tissue particularly vulnerable. Their remarkable evolutionary association, perhaps based upon a potential for
Fig. 3 Northern blot analysis of $H s c B$. RNAs from the human tissues indicated on the top were loaded. High stringency hybridization with the $H s c B$ probe was followed by hybridization with a $\beta$-actin probe to demonstrate uniformity of RNA loading. Positions of the RNA standard markers are indicated

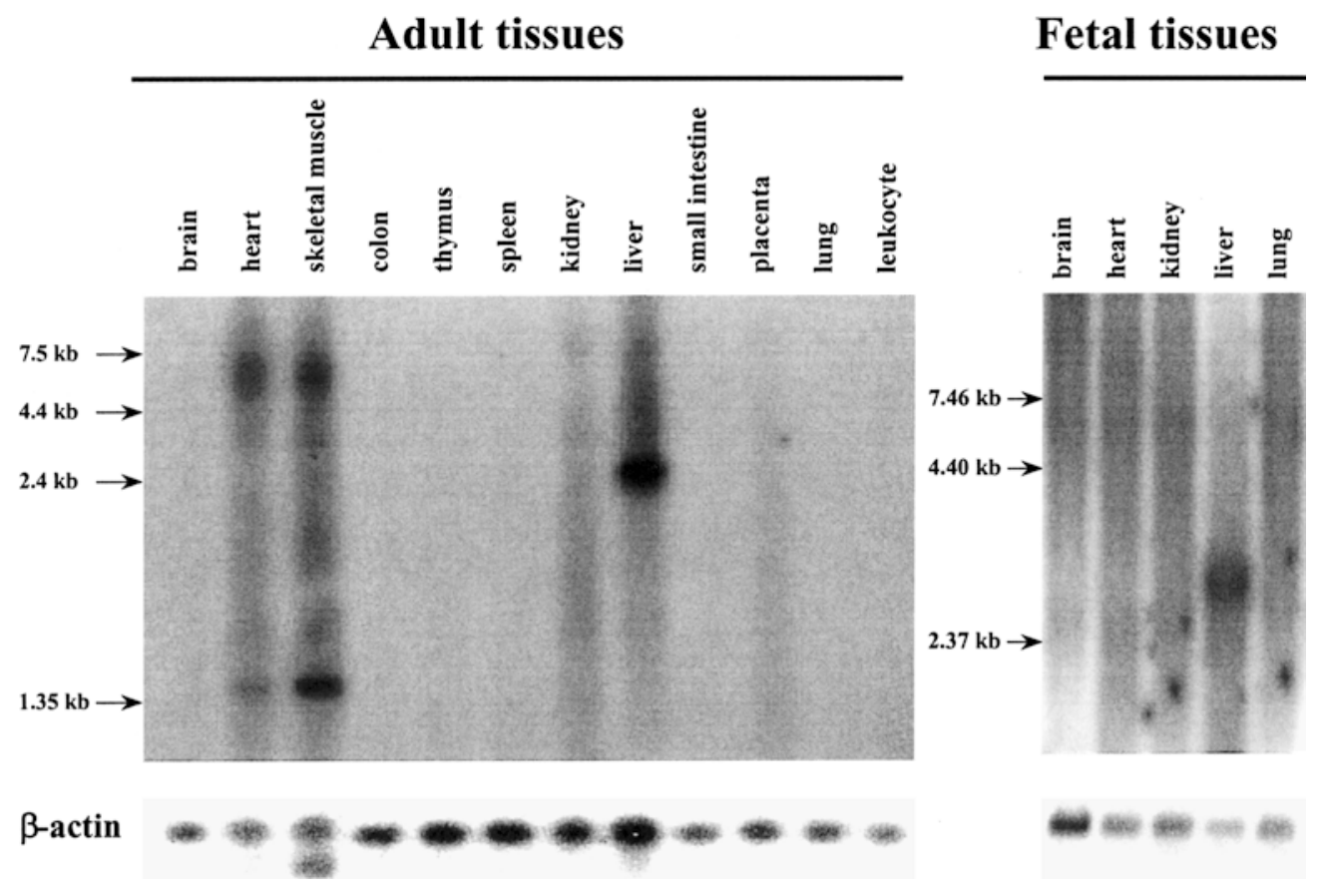


functional and/or physical interactions in $\mathrm{Fe}-\mathrm{S}$ cluster formation, makes $H s c B$ a promising candidate gene for evaluation in hereditary ataxia syndromes. This is particularly intriguing from the perspective of ataxia pathogenesis, since in a screen for second-site suppressors of polyglutamine toxicity, an important but still incompletely explained pathogenic mechanism underlying several dominant ataxia syndromes (Ross 2002; Cummings and Zoghbi 2000), the two different suppressor genes recognized encoded $\mathbf{J}$ proteins (Kazemi-Esfarjani and Benzer 2000). This suggests that defects in J-protein mechanisms might predispose to pathogenic processes leading to an intermediate shared with polyglutamine toxicity, both thereby producing susceptibility to ataxia (Opal and Zoghbi 2002). Demonstration of a direct interaction of $\mathrm{HscB}$ with frataxin, a shared functional role in Fe-S cluster formation, or in suppressing polyglutamine toxicity would further strengthen the functional candidacy of $\mathrm{HscB}$ in the spectrum of mitochondriarelated disease phenotypes observed in Friedreich ataxia and the polyglutamine-expansion diseases, including cardiomyopathy, neuropathy, diabetes mellitus and ataxia itself.

Acknowledgements This work was supported by NIH grants MH59222 and HD035458 (to J.J.G.) and GM54264 (to L.E.V.).

\section{References}

Bekri S, Kispal G, Lange H, Fitzsimons E, Tolmie J, Lill R, Bishop DF (2000) Human ABC7 transporter: gene structure and mutation causing X-linked sideroblastic anemia with ataxia with disruption of cytosolic iron-sulfur protein maturation. Blood 96:3256-3264

Campuzano V, Montermini L, Molto MD, Pianese L, Cossee M, Cavalcanti F, Monros E, Rodius F, Duclos F, Monticelli A, et al. (1996) Friedreich's ataxia: autosomal recessive disease caused by an intronic GAA triplet repeat expansion. Science 271:1423-1427

Cavadini P, O'Neill HA, Benada O, Isaya G (2002) Assembly and iron-binding properties of human frataxin, the protein deficient in Friedreich ataxia. Hum Mol Genet 11:217-227

Cummings CJ, Zoghbi HY (2000) Trinucleotide repeats: mechanisms and pathophysiology. Annu Rev Genomics Hum Genet $1: 281-328$

Cupp-Vickery JR, Vickery LE (2000) Crystal structure of Hsc20, a J-type co-chaperone from Escherichia coli. J Mol Biol 304:835845

Dhe-Paganon S, Shigeta R, Chi YI, Ristow M, Shoelson SE (2000) Crystal structure of human frataxin. J Biol Chem 275:3075330756

Hoff KG, Silberg, JJ, Vickery LE (2000) Interaction of the ironsulfur cluster assembly protein IscU with the Hsc66/Hsc20 molecular chaperone system in Escherichia coli. Proc Natl Acad Sci USA 97:7790-7795

Hoff KG, Ta, DT, Tapley TL, Silberg JJ, Vickery LE (2002) Hsc66 substrate specificity is directed toward a discreet region of the iron-sulfur cluster template protein IscU. J Biol Chem 277: 27353-27359
Huynen MA, Snel B, Bork P, Gibson TJ (2001) The phylogenetic distribution of frataxin indicates a role in iron-sulfur cluster protein assembly. Hum Mol Genet 10:2463-2468

Kaplan J (2002) Spinocerebellar ataxias due to mitochondrial defects. Neurochem Intl 40:553-557

Kawula TH, Lelivelt MJ (1994) Mutations in a gene encoding a new Hsp70 suppress rapid DNA inversion and $b g l$ activation, but not proU derepression, in hns-1 mutant Escherichia coli. J Bacteriol 176:610-619

Kazemi-Esfarjani P, Benzer S (2000) Genetic suppression of polyglutamine toxicity in Drosophila. Science 287:1837-1840

Kelley WL (1998) The J-domain family and the recruitment of chaperone power. Trends Biochem Sci. 23:222-227

Kim R, Saxena S, Gordon DM, Pain D, Dancis A (2001) J-domain protein, Jaclp, of yeast mitochondria required for iron homeostasis and activity of Fe-S cluster proteins. J Biol Chem 276:17524-17532

Lutz T, Westermann B, Neupert W, Herrmann JM (2001) The mitochondrial proteins Ssq1 and Jac1 are required for the assembly of iron sulfur clusters in mitochondria. J Mol Biol 307:815-825

Matsuura T, Yamagata T, Burgess D L, Rasmussen A, Grewal R $\mathrm{P}$, Watase K, Khajavi M, McCall AE, Davis CF, Zu L, et al. (2000) Large expansion of the ATTCT pentanucleotide repeat in spinocerebellar ataxia type 10. Nat Genet 26:191-194

Muhlenhoff U, Richhardt N, Ristow M, Kispal G, Lill R (2002) The yeast frataxin homolog Yfhlp plays a specific role in the maturation of cellular Fe/S proteins. Hum Mol Genet 11:20252036

Nishino I, Spinazzola A, Hirano M (1999) Thymidine phosphorylase gene mutations in MNGIE, a human mitochondrial disorder. Science 283:689-692

Opal P, Zoghbi HY (2002) The role of chaperones in polyglutamine disease. Trends Mol Med 8:232-236

Patel PI, Isaya G (2001) Friedreich ataxia: from GAA triplet-repeat expansion to frataxin deficiency. Am. J Hum Genet 69:15-24

Pfanner N (2000) Protein sorting: recognizing mitochondrial presequences. Curr Biol 10:R412-R415

Pfanner N, Wiedemann N (2002) Mitochondrial protein import: two membranes, three translocases. Curr Opin Cell Biol 14:400 411

Ross CA (2002) Polyglutamine pathogenesis: emergence of unifying mechanisms for Huntington's disease and related disorders. Neuron 35:819-822

Seaton BL, Vickery LE (1994) A gene encoding a DnaK/hsp70 homolog in Escherichia coli. Proc Natl Acad Sci USA. 91:20662070

Silberg JJ, Hoff KG, Tapley TL, Vickery LE (2001) The Fe/S assembly protein IscU behaves as a substrate for the molecular chaperone Hsc66 from Escherichia coli. J Biol Chem 276:16961700

Strain J, Lorenz CR, Bode J, Garland S, Smolen GA, Ta DT, Vickery LE, Culotta VC (1998) Suppression of superoxide dismutase (SOD1) deficiency in Saccharomyces cerevisiae. Identification of proteins predicted to mediate iron sulfur cluster assembly. J Biol Chem 273:31138-31144

Vickery LE, Silberg, JJ, Ta DT (1997) Hsc66 and Hsc20, a new, heat shock cognate molecular chaperone system from Escherichia coli. Protein Sci 6:1047-1056

Voisine C, Cheng YC, Ohlson M, Schilke B, Hoff K, Beinert H, Marszalek J, Craig EA (2001) Jac1, a mitochondrial J-type chaperone, is involved in the biogenesis of $\mathrm{Fe} / \mathrm{S}$ clusters in Saccharomyces cerevisiae. Proc Natl Acad Sci USA 98:14831488 